\title{
Unbiased Vessel-Diameter Quantification based on the FWHM Criterion
}

\author{
Henri Bouma $^{a}$, Javier Oliván Bescós ${ }^{b}$, Anna Vilanova $^{a}$ and Frans A. Gerritsen ${ }^{a, b}$ \\ ${ }^{a}$ Technische Universiteit Eindhoven, P.O. Box 513, 5600 MB Eindhoven, The Netherlands; \\ ${ }^{b}$ Philips Medical Systems, P.O. Box 10000, 5680 DA Best, The Netherlands.
}

\begin{abstract}
The full-width at half-max (FWHM) criterion is often used for both manual and automatic quantification of the vessel diameter in medical images. The FWHM criterion is easy to understand and it can be implemented with low computational cost. However, it is well known that the FWHM criterion can give an over- and underestimation of the vessel diameter. In this paper, we propose a simple and original method to create an unbiased estimation of the vessel diameter based on the FWHM criterion and we compared the robustness to noise of several edge detectors. The quantitative results of our experiments show that the proposed method is more accurate and precise than other (more complex) edge detectors, even for small vessels.
\end{abstract}

Keywords: Segmentation

\section{INTRODUCTION}

Quantification of the local vessel diameter, or the cross-sectional vessel area, is essential for the correct diagnosis of vascular diseases. For example, the relative decrease in diameter of a stenosis is an important factor in determining the treatment therapy. ${ }^{1}$ However, inherent to image acquisition is a blurring effect, which can be modelled by the convolution with a point-spread function (PSF). This blurring function causes conventional methods $^{2-4}$ to inaccurately locate the vessel boundary, leading to a bias in most vessel-diameter quantification methods. ${ }^{5-7}$

Recently, Manniesing ${ }^{8}$ proposed a method to reduce the average bias over vessels with different diameters. However, the bias - which depends on the diameter - was not shown for each of the vessels separately. Mendonça ${ }^{9}$ and Bouma ${ }^{10}$ proposed a method to remove the bias caused by derivative-based edge detectors. However, the computation of nine image derivatives is expensive in comparison to intensity based methods, such as the FWHM criterion. ${ }^{11-13}$ The FWHM criterion is often used because it gives a reproducible and precise - although not accurate due to bias - estimation of the vessel diameter.

In this paper, we propose a simple method based on FWHM that removes the bias for vessels at low computational cost. We will analyze the signal-to-noise ratio of several edge detectors and show that the FWHM is not only cheaper but also more robust to noise than the derivative-based edge detectors. Finally, the proposed method will be compared with the edge-detection methods - such as the maximum gradient magnitude, Marr-Hildreth ${ }^{14}$ and Canny ${ }^{3}$ - on synthetic data and on computed-tomography (CT) data of a phantom.

\section{METHOD}

Grey-level thresholding is a fast operation that is still widely used for image segmentation. ${ }^{15}$ A bright object with a high intensity $I_{H}$ can be separated from a dark background with a low intensity $I_{L}$ by using a threshold at $I_{t h r}=\left(I_{H}+I_{L}\right) / 2$. For large objects with straight edges, this intensity indicates the correct location of the separation between two objects.

Some methods use one iso-value - which is fixed at half the intensity of the object before blurring - for segmentation and visualization ${ }^{16}$ of vessels. However, a global threshold based on this value shows a large underestimation of the diameter for small vessels, and the vessel boundary may even be missed while it is still clearly visible in the images.

Henri Bouma recently joined TNO in The Hague, henri.bouma@tno.nl, phone +31.70.37.40399

Medical Imaging 2007: Image Processing, edited by Josien P. W. Pluim, Joseph M. Reinhardt, Proc. of SPIE Vol. 6512, 65122N, (2007) · 1605-7422/07/\$18 - doi: 10.1117/12.709273 
Other methods use the FWHM criterion to determine the vessel diameter. This criterion adapts the threshold to the local maximum and minimum intensity on the edge transition. As the maximum intensity of a small vessel decreases due to blurring, the threshold will also decrease. However, this method also shows a large dislocation for small vessels.

In this paper, we propose an unbiased vessel-diameter quantification method based on FWHM. Figure 1 shows the dislocation $\left(r_{0}-R\right)$ of the vessel with true radius $R$ and a detected radius $r_{0}$ for the thresholding methods based on an iso-value or on the FWHM criterion. The analysis is based on the assumptions that the vessel has a circular cross-section and that it was Gaussian blurred with standard deviation $\sigma$ during acquisition. Although the point-spread function (PSF) is not completely Gaussian, this approximation can be made for several imaging modalities, including CT. ${ }^{17}$ White Gaussian noise was used to analyze the robustness of the method. To verify the validity of the assumptions, the method was used on CT data.

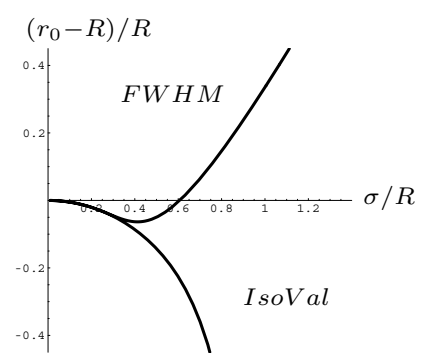

Figure 1. The dislocation $\left(r_{0}-R\right)$ of the vessel boundary for two threshold-based methods. The fixed iso-value segmentation shows an underestimation, and the FWHM criterion shows both an under- and overestimation of the vessel with radius $R$ and Gaussian blur with standard deviation $\sigma$.

\subsection{Method for Unbiased Quantification based on FWHM}

The gradient magnitude $\left(L_{w}\right)$ of a disk, with intensity $I_{H}=1.0$ inside and $I_{L}=0.0$ outside the disk, at distance $r$ from the center is: ${ }^{10}$

$$
L_{w}(r, R, \sigma)=\frac{R}{\sigma^{2}} \exp \left(-\frac{r^{2}+R^{2}}{2 \sigma^{2}}\right) I_{1}\left(\frac{r R}{\sigma^{2}}\right)
$$

Where $I_{1}$ is the modified Bessel function of the first kind. With this equation, we can compute numerically the intensity $L$, the location of the thresholds and the relation between the detected radius $r_{0}$ and the true radius $R$ of a cylindrical vessel. The relation in Figure 2 allows a correction by mapping the dislocated 'input' to the corrected 'output' radius, and it can be implemented as a look-up-table operation. Diminishing the bias leads to a more accurate measurement.

The stability of this mapping is analyzed by looking at the effects on the output of a perturbation on the input. Figure 3 shows that a perturbation of $2 \%$ on the detected input radius will lead to a small perturbation on the output (less than 10\%), when $R>0.7 \sigma\left(r_{0}>1.23 \sigma\right)$. This is small in comparison to the error of the uncorrected radius (Fig. 3b: dashed). For very small vessels (e.g., $R<0.7 \sigma$ ), the precision can be increased by averaging multiple measurements along the vessel.

\subsection{Analysis of the Robustness to Noise}

To analyze the robustness to noise of several edge detectors, the signal-to-noise ratio (SNR) is derived for a Gaussian blurred image ( $L$, which is used for FWHM), gradient magnitude $\left(L_{w}\right)$, the Laplacian $(\Delta L$, which is used by Marr-Hildreth) and the second-order derivative in the gradient direction ( $L_{w w}$, which is used by Canny). Our analysis will focus on an image with zero-mean white Gaussian noise on the input with standard deviation $\sigma_{n i}$. The SNR in decibel is defined as:

$$
S N R_{[d B]}=20 \log _{10}\left(\frac{\mu_{s}}{\sigma_{n}}\right)
$$




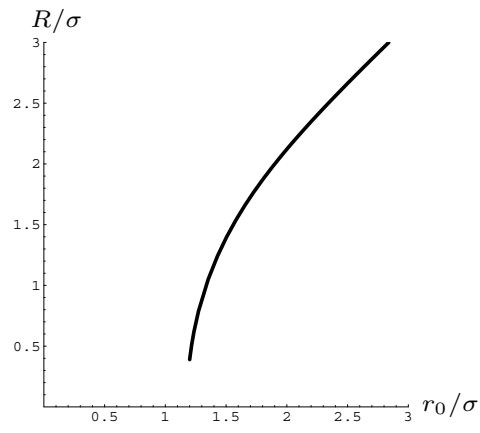

(a)

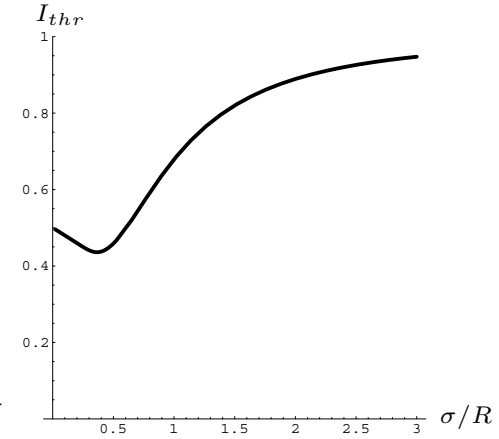

(b)

Figure 2. (a) The relation between the true radius $R$ of the vessel and the radius detected with FWHM $r_{0}$ after Gaussian blur with standard deviation $\sigma$ and (b) the relation between the true radius and the threshold $I_{t h r}$ for a corrected segmentation of the vessel. These corrections of the FWHM criterion diminish the bias from the radius estimation.

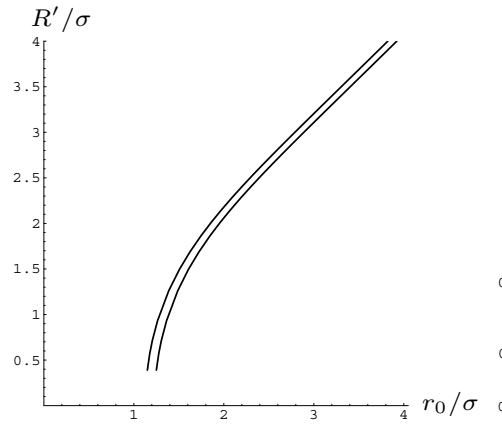

(a)

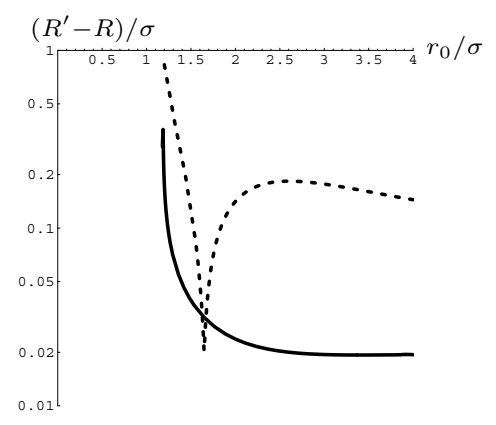

(b)

Figure 3. The effects of $2 \%$ perturbation on the radius detected with the FWHM $r_{0}$ on the corrected radius $R^{\prime}$. (a) The two curves indicate the perturbation on the estimated radius $R^{\prime}$. (b) dashed: The sum of systematic and stochastic errors in the uncorrected FWHM radius and (b) solid: the stochastic error in the estimation of the corrected method. 
where $\mu_{s}$ is the signal amplitude and $\sigma_{n}$ is the standard deviation of the noise. First, the computation of $\sigma_{n}$ will be discussed for several edge detectors, and after that the computation of $\mu_{s}$.

\subsubsection{Noise Distribution of Edge Detectors in 2D}

The relation between the standard deviation of the noise before $\left(\sigma_{n i}\right)$ and after $\left(\sigma_{n o}\right)$ filtering with a FIR-based kernel $K$ is: ${ }^{18}$

$$
\left(\frac{\sigma_{n o}}{\sigma_{n i}}\right)^{2}=\int_{-\infty}^{\infty}(K)^{2} d \vec{x}
$$

Four FIR-based kernels that will be used in our analysis of noise in a two-dimensional (2D) image are the zeroth-, first- and second-order Gaussian derivatives in the $x$-direction $\left(L, L_{x}\right.$ and $\left.L_{x x}\right)$ and the Laplacian $\left(\Delta L=L_{x x}+L_{y y}\right)$. The relation between input and output noise is:

$$
\begin{aligned}
\sigma_{L}^{2} & =\frac{1}{4 \pi \sigma_{o p}^{2}} \sigma_{n i}^{2} & \sigma_{L x}^{2} & =\frac{1}{8 \pi \sigma_{o p}^{4}} \sigma_{n i}^{2} \\
\sigma_{L x x}^{2} & =\frac{3}{16 \pi \sigma_{o p}^{6}} \sigma_{n i}^{2} & \sigma_{\Delta L}^{2} & =\frac{1}{2 \pi \sigma_{o p}^{6}} \sigma_{n i}^{2}
\end{aligned}
$$

where $\sigma_{o p}$ is the standard deviation of the Gaussian operator.

The gradient magnitude $L_{w}=\sqrt{L_{x}^{2}+L_{y}^{2}}$ cannot be computed as a finite-impulse response (FIR) filtering operation. Although, $L_{w}$ is a rotated version of $L_{x}$, we cannot simply assume that the distribution of $L_{w}$ is equal to that of $L_{x}$, because the rotation of $L_{w}$ is dependent on $L_{x}$. Therefore, we will first compute the distributions of $L_{x}^{2}$ and $L_{w}^{2}$ (the distribution of $L_{y}^{2}$ is of course identical to $L_{x}^{2}$ ). The probability density function (P) of $L_{x}^{2}$ can be computed through the cumulative distribution function (C):

$$
\begin{aligned}
\mathrm{C}_{L x 2}(x) & =\int_{-\sqrt{x}}^{\sqrt{x}} \mathcal{G}\left(y-0, \sigma_{L x}\right) \mathrm{d} y \\
\mathrm{P}_{L x 2}(x) & = \begin{cases}\frac{\partial C_{L x 2}(x)}{\partial x}=\frac{1}{\sigma_{L x} \sqrt{2 \pi x}} \exp \left(-\frac{x}{2 \sigma_{L x}^{2}}\right), & x \geq 0 \\
0, & x<0\end{cases}
\end{aligned}
$$

We assume that the covariance between $L_{x}^{2}$ and $L_{y}^{2}$ can be neglected on an image with white Gaussian noise. The density $P_{L w 2}$ can be computed as a convolution between $P_{L x 2}$ and $P_{L y 2}$.

$$
P_{L w 2}(x)=\int_{0}^{x} P_{L x 2}(y) P_{L y 2}(x-y) \mathrm{d} y=\frac{1}{2 \sigma_{L x}^{2}} \exp \left(-\frac{x}{2 \sigma_{L x}^{2}}\right), \quad x \geq 0
$$

Finally, the function $P_{L w}$ is computed through the distribution $C$,

$$
\begin{aligned}
\mathrm{C}_{L w}(x) & =\int_{0}^{x^{2}} P_{L w 2}(y) \mathrm{d} y \\
\mathrm{P}_{L w}(x) & =\frac{x}{\sigma_{L x}^{2}} \exp \left(-\frac{x^{2}}{2 \sigma_{L x}^{2}}\right), \quad x \geq 0
\end{aligned}
$$

resulting in:

$$
\begin{aligned}
\mu_{L w} & =\int P_{L w}(x) x \mathrm{~d} x=\sqrt{\frac{\pi}{2}} \sigma_{L x}=\frac{1}{4 \sigma_{o p}^{2}} \sigma_{n i} \\
\sigma_{L w}^{2} & =\int P_{L w}(x)(x-\mu)^{2} \mathrm{~d} x=\frac{4-\pi}{2} \sigma_{L x}^{2}=\frac{4-\pi}{16 \pi \sigma_{o p}^{4}} \sigma_{n i}^{2}
\end{aligned}
$$


The second-order derivative in the gradient direction $L_{w w}$ is a rotated version of $L_{x x} . L_{w w}$ is rotated by $L_{x}$ and $L_{y}$, and it is independent of $L_{x x}$. Therefore, we can assume that its variance is equal to that of $L_{x x}$ on white Gaussian noise.

$$
\sigma_{L w w}^{2}=\frac{3}{16 \pi \sigma_{o p}^{6}} \sigma_{n i}^{2}
$$

These equations were experimentally verified (Sec. 3.1). As shown by the equations, the variance of noise $\sigma_{\text {no }}^{2}$ can be made scale invariant with a factor $\sigma_{o p}^{2 n+2}$, where $n$ is the maximum order of the derivatives (so $n$ can be $0(L), 1\left(L_{w}\right)$ or $\left.2\left(L_{w w}, \Delta L\right)\right)$.

\subsubsection{Noise distribution of edge detectors in $3 D$}

The variance after filtering for three-dimensional (3D) images is computed in the same way, resulting in:

$$
\begin{array}{rlrl}
\sigma_{L}^{2} & =\frac{1}{8 \pi^{3 / 2} \sigma_{o p}^{3}} \sigma_{n i}^{2} & \sigma_{\Delta L}^{2} & =\frac{15}{32 \pi^{3 / 2} \sigma_{o p}^{7}} \sigma_{n i}^{2} \\
\sigma_{L_{w}}^{2} & =\frac{3 \pi-8}{16 \pi^{5 / 2} \sigma_{o p}^{5}} \sigma_{n i}^{2} & \mu_{L_{w}}=\frac{1}{\sqrt{2} \pi^{5 / 4} \sigma_{o p}^{5 / 2}} \sigma_{n i} \\
\sigma_{L_{w w}}^{2} & =\frac{3}{32 \pi^{3 / 2} \sigma_{o p}^{7}} \sigma_{n i}^{2} & &
\end{array}
$$

The variance of noise $\sigma_{n o}^{2}$ in $3 \mathrm{D}$ decreases faster than in $2 \mathrm{D}$. To make $\sigma_{n o}^{2}$ scale invariant, a factor $\sigma_{o p}^{2 n+3}$ is needed, instead of $\sigma_{o p}^{2 n+2}$.

\subsubsection{Signal amplitude of edge detectors}

A disk in a two-dimensional image with intensity $I_{H}=1.0$ inside and $I_{L}=0.0$ outside the disk has the following equations ${ }^{10}$ for $L_{w w}$ and $\Delta L$ :

$$
\begin{aligned}
L_{w w}(r, R, \sigma) & =e^{-\frac{r^{2}+R^{2}}{2 \sigma^{2}}}\left(-\frac{R^{2}}{\sigma^{4}} I_{0}\left(\frac{r R}{\sigma^{2}}\right)+\left(\frac{r R}{\sigma^{4}}+\frac{R}{r \sigma^{2}}\right) I_{1}\left(\frac{r R}{\sigma^{2}}\right)\right) \\
\Delta L(r, R, \sigma) & =e^{-\frac{r^{2}+R^{2}}{2 \sigma^{2}}}\left(-\frac{R^{2}}{\sigma^{4}} I_{0}\left(\frac{r R}{\sigma^{2}}\right)+\frac{r R}{\sigma^{4}} I_{1}\left(\frac{r R}{\sigma^{2}}\right)\right)
\end{aligned}
$$

where $R$ is the radius of the disk, $r$ is the distance to the center, and $I_{n}$ is the modified Bessel function of the first kind. From $L_{w}$ (Eq. 1), the intensity $L$ can be computed.

The amplitude of a signal is defined as the distance between the maximum absolute peak value and the mean of the signal (which is zero).

The amplitude of $L$ on a disk is always located at the center of the disk.

$$
L(0, R, \sigma)=1-\exp \left(-\frac{R^{2}}{2 \sigma^{2}}\right)
$$

For $R<2 \sigma$, the maximum of $L_{w w}$ and $\Delta L$ is also located at the center of the disk.

$$
\begin{aligned}
L_{w w}(0, r, \sigma) & =-\frac{R^{2}}{2 \sigma^{4}} \exp \left(-\frac{R^{2}}{2 \sigma^{2}}\right) \\
\Delta L(0, r, \sigma) & =-\frac{R^{2}}{\sigma^{4}} \exp \left(-\frac{R^{2}}{2 \sigma^{2}}\right)
\end{aligned}
$$

Other amplitudes can be computed numerically with the Equations 1, 13 and 14.

Differential operators are not scale-invariant. This means that the slope of a blurred signal will decrease as the amount of blurring increases. If we consider the transformation $x / \sigma \rightarrow \tilde{x}$, then $\tilde{x}$ is dimensionless and the operator is scale-invariant. The dimensionless coordinate is called the natural coordinate, ${ }^{19}$ which has a scaling factor: $\frac{\partial^{n}}{\partial x^{n}} \rightarrow \sigma^{n} \frac{\partial^{n}}{\partial \tilde{x}^{n}}$. The scaling factor avoids the decrease of the amplitude of the signal at a larger scale. 


\subsubsection{SNR of edge detectors}

As mentioned before, the signal amplitude $\mu_{s}$ can be made scale invariant with a factor $\sigma_{o p}^{n}$ and the standard deviation of noise $\sigma_{n o}$ can be made scale invariant with a factor $\sigma_{o p}^{n+1}$ in $2 \mathrm{D}$ and $\sigma_{o p}^{n+3 / 2}$ in $3 \mathrm{D}$. So, to make the signal-to-noise ratio scale invariant a factor $\sigma_{o p}$ is needed in $2 \mathrm{D}$, and $\sigma_{o p}^{3 / 2}$ in $3 \mathrm{D}$.

Figure 4 shows the SNR for these methods near the edge of a cylindrical vessel when computed in 3D data or in $2 \mathrm{D}$ cross sections. The figure shows that FWHM $(L)$ can obtain a higher SNR at a blurring scale that is (almost) twice as low (or fine) as that of the others. The low scale has the advantage that it avoids an extra bias and the interference with neighboring objects. The high SNR leads to a precise measurement and it makes the FWHM method more robust to noise than the other edge detectors.

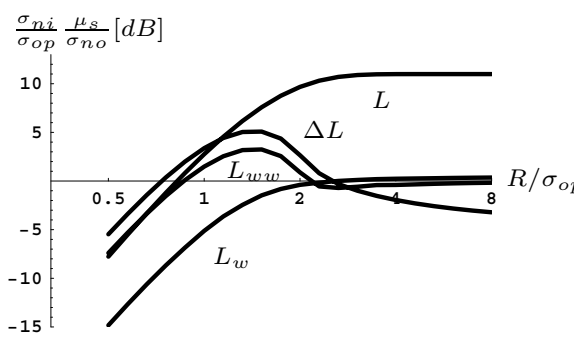

$2 \mathrm{D}$

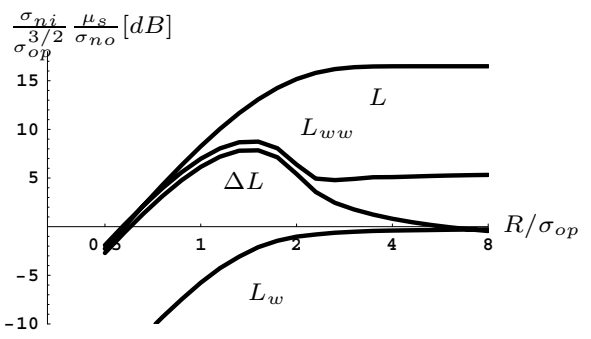

$3 \mathrm{D}$

Figure 4. The signal-to-noise ratio $\left(\mu_{s} / \sigma_{n o}\right)$ in decibel for several operators $\left(L, L_{w}, L_{w w}\right.$ and $\left.\Delta L\right)$ based on Gaussian derivatives with standard deviation $\sigma_{o p}$ in an image with white Gaussian noise $\left(\sigma_{n i}\right)$ that contains a disk with radius $R$.

As an example, Figure 5 shows the response of FWHM, which is based on $L$, and the derivative-based edge detectors to a disk in a noisy 2D image. The figure shows that FWHM has a stronger response than the others.

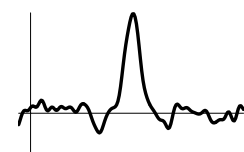

$L$

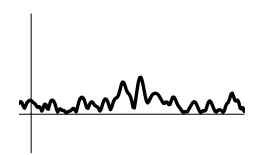

$L_{w}$

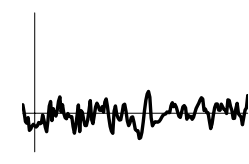

$L_{w w}$

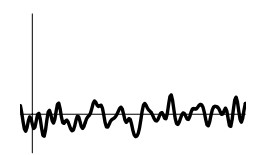

$\Delta L$

Figure 5. Example of cross-sectional responses of Gaussian-derivative operators $(\sigma=2.5 \mathrm{px})$ to a disk $(R=7.0 \mathrm{px})$ in a noisy $2 \mathrm{D}$ image $\left(S N R_{i}=0 \mathrm{~dB}\right)$. The figure shows that FWHM, which is based on $L$, has a stronger response than the derivative-based edge detectors.

\section{EXPERIMENTS AND RESULTS}

Three experiments were performed to analyze the proposed method quantitatively. In the first experiment, we used noisy synthetic data to verify the derivation of the noise distributions. In the second and third experiment, the accuracy and precision of the corrected FWHM criterion was validated. In the second experiment we used noisy synthetic data with cylindrical structures and in the third experiment we used computed-tomography (CT) image of a phantom.

\subsection{Noise Distributions on Synthetic Data}

For the derivation of the noise distribution (Sec. 2.2), we assumed independence for some operations to estimate the mean and the variance of an image after applying an edge detector. With this experiment we verified whether the assumptions are valid for images that contain white Gaussian noise. 
The experiment was performed on a 2D image of 300x300 pixels and on a 3D image of 50x50x50 voxels. Both images contained white Gaussian noise with zero mean and $\sigma_{n i}=1$. Gaussian operators were used, with an exponentially sampled $\sigma_{o p}$ in the range from 2.0 to 16.0 pixels in 7 steps. For the operators $L, L_{w}, L_{w w}$ and $\Delta L$ the output variance $\sigma_{n o}^{2}$ was measured, and for $L_{w}$ the mean $\mu_{L w}$ was also measured.

In $2 \mathrm{D}$, the correlation between $\log \left(\sigma_{o p}\right)$ and $\log \left(\sigma_{n o}^{-1 / n}\right)$ was larger than 0.999 for all operators. The correlation between $\log \left(\sigma_{o p}\right)$ and $\log \left(\mu_{L w}^{-1 / n}\right)$ was also larger than 0.999 . The root-mean-square (RMS) error in estimating the values of Table 1 for $2 \mathrm{D}$ was on average 0.06 .

In 3D, the correlation between $\log \left(\sigma_{o p}\right)$ and $\log \left(\sigma_{n o}^{-2 /(2 n+1)}\right)$ was larger than 0.999 for all operators, and the correlation between $\log \left(\sigma_{o p}\right)$ and $\log \left(\mu_{L w}^{-2 /(2 n+1)}\right)$ was also larger than 0.999. The RMS error in estimating the values of Table 1 for $3 \mathrm{D}$ was on average 0.07 .

These results show that the derived distributions accurately predict the measured distributions.

Table 1. The $R M S$ error in estimating the distribution values that were predicted by the equations in Sec. 2.2 .

\begin{tabular}{|c||c|c|c||c|c|c|}
\hline \multicolumn{1}{|c||}{} & \multicolumn{3}{c||}{$2 \mathrm{D}$} & \multicolumn{3}{c|}{$3 \mathrm{D}$} \\
\cline { 2 - 7 } & Eq. & value & RMS & Eq. & value & RMS \\
\hline \hline$\sigma_{L}$ & 4 & $4 \pi$ & 0.11 & 10 & $8 \pi^{3 / 2}$ & 0.11 \\
$\sigma_{\Delta L}$ & 4 & $2 \pi$ & 0.04 & 10 & $32 \pi^{3 / 2} / 15$ & 0.08 \\
$\mu_{L w}$ & 8 & 4 & 0.03 & 11 & $\sqrt{2} \pi^{5 / 4}$ & 0.05 \\
$\sigma_{L w}$ & 8 & $16 \pi /(4-\pi)$ & 0.06 & 11 & $16 \pi^{5 / 2} /(3 \pi-8)$ & 0.06 \\
$\sigma_{L w w}$ & 9 & $16 \pi / 3$ & 0.04 & 12 & $32 \pi^{3 / 2} / 3$ & 0.04 \\
\hline
\end{tabular}

\subsection{Unbiased FWHM on Synthetic Data}

To verify the theory presented in this paper and to analyze the robustness of our method, noisy synthetic data was created that contained cylinders as a vessel model (Fig. 6a). The intensities in the synthetic images were 1.0 inside the unblurred cylinders and 0.0 outside, and the radii of the cylinders were in the range from 1.6 to 5.8 pixels. The standard deviation of the PSF $\sigma_{p s f}$ was 1.5 and 2.2 pixels in $x y$ - and $z$-directions respectively. White Gaussian noise was added with standard deviation $\sigma_{\text {noise }}=0.085$ (which is equivalent to a signal-to-noise ratio of 21 and 9.4 decibel for large and small vessels respectively). Some extra blurring $\sigma_{o p}$ was added in the $x y$-direction to make the total blurring isotropic with $\sigma_{t o t}=\sqrt{\sigma_{p s f}^{2}+\sigma_{o p}^{2}}=2.2$ pixels.

The half-max radius was computed for each cross-section as an average of the radii in 32 directions. The proposed method was applied for each cross section to remove the bias. For each vessel with a different radius, the mean $\mu$ and standard deviation $\sigma$ of both the uncorrected and corrected radius were computed over 100 cross sections.

The dislocations are summarized in Figure 6b, where the dashed curves show the stochastic errors.

For the smallest cylinders of only 1.6 pixels, the error of the uncorrected FWHM method was larger than three times the stochastic error of the unbiased method. From this we can conclude that, even for small cylinders, the unbiased method is the most accurate in more than $99 \%$ of the measurements. The precision can easily be increased by adding extra blur in the direction along the vessel or - as mentioned before - by averaging multiple measurements along the vessel.

The results show that the proposed method diminishes the bias from the diameter estimation.

\subsection{Unbiased FWHM on CT Data}

In order to show that our method can be applied to CT data, and to perform quantitative measurements, a threedimensional cerebrovascular phantom ${ }^{20}$ was scanned of which the size is accurately known (Fig. 7a). The labels and the diameters of the arteries represented in the phantom are: posterior communicating (PcomA, 1.0mm), posterior-cerebral (PCA, 2.00mm), middle-cerebral (MCA, 2.78mm) and internal carotid (ICA, 3.50mm) arteries. In the reconstructed volume, the voxel size was 0.3125 and $0.30 \mathrm{~mm}$ in $x y$ - and $z$-directions respectively, with 


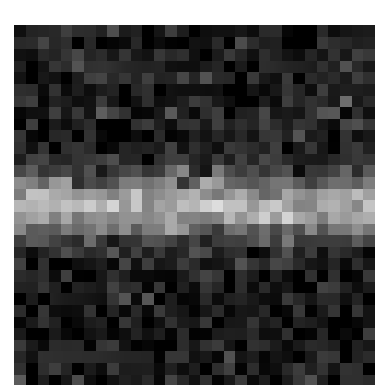

(a)

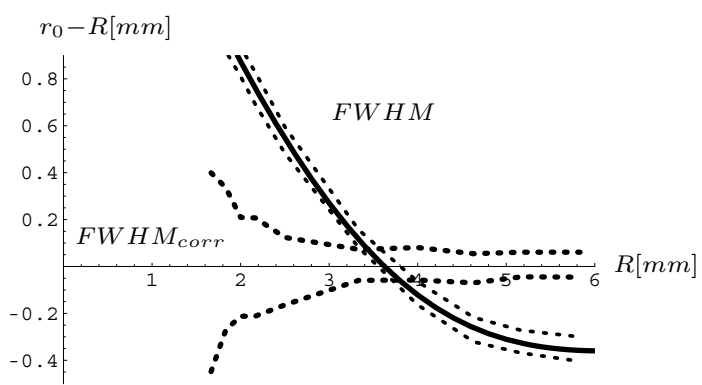

(b)

Figure 6. (a) Slice of a synthetic image that contains a cylinder with a radius of $R=1.8$ pixels. (b) The dislocation $\left(r_{0}-R\right)$ of the vessel boundary for the biased and the corrected FWHM criterion for several vessels with radius $R$ (mm). The stochastic errors $(\mu-\sigma$ and $\mu+\sigma)$ are indicated with the dashed curves and the theoretic bias of the uncorrected FWHM criterion is indicated by the solid curve.

a slice thickness of $1.5 \mathrm{~mm}$. The standard deviation of the PSF was 0.47 and $0.66 \mathrm{~mm}$ in $x y$ - and $z$-direction respectively. Some extra blurring was added in the $x y$-direction to remove noise and to make the total blurring isotropic.

The measurements were performed in the following way. Two points were selected manually on each of the mentioned vessels. The points were centered with the center of mass of the FWHM region in a cross-sectional plane. The orientation of the plane was based on the structure tensor. ${ }^{21}$ Each of the edge detectors was used to detect the vessel boundary in this plane, and the area of the polygon through the contour points was used to estimate the average diameter. The diameter was measured over $4.0 \mathrm{~cm}$ in 40 steps and these measurements were used to compute the mean and the standard deviation.

The dislocations are summarized in Figure $7 \mathrm{~b}$. The results show that the proposed method is able to remove the bias for all vessels in a 10\% error range, while others obtain an overestimation of more than $100 \%$.

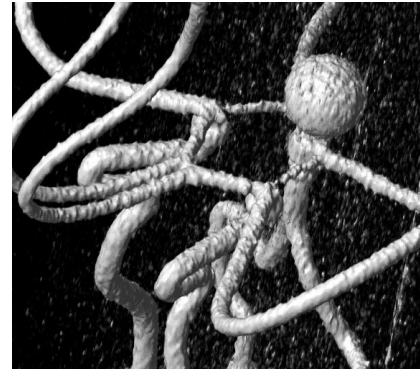

(a)

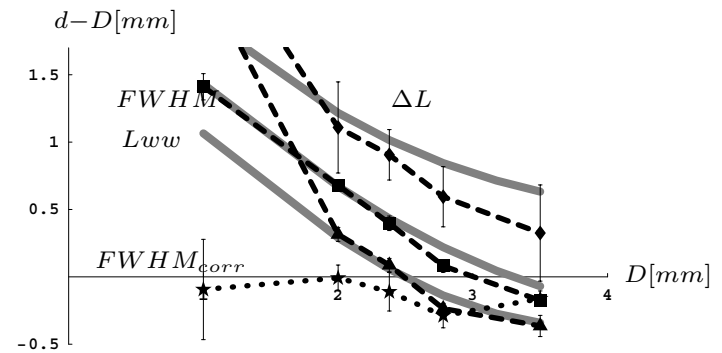

(b)

Figure 7. (a) Surface rendering of the CT data of the phantom. (b) The dislocation $\left(d_{0}-D\right)$ of the vessel boundary for Marr-Hildreth $(\Delta L)$, FWHM, Canny $\left(L_{w w}\right)$ and corrected FWHM for several vessels with diameter $D$ (mm). The theoretical curves are shown in grey.

\section{CONCLUSIONS}

In this paper, we proposed a new method for unbiased vessel-diameter quantification based on the FWHM criterion at low computational cost. We analyzed the SNR of several edge detectors on cylindrical structures and we showed that the FWHM is more robust to noise than the derivative-based edge detectors. The quantitative results obtained with synthetic and CT images showed that the proposed method is accurate and precise, even for vessels with a radius smaller than the point-spread function. 


\section{ACKNOWLEDGMENTS}

The authors thank Annet Waaijer of the Universitair Medisch Centrum in Utrecht for providing the CT data of the phantom.

\section{REFERENCES}

1. A. F. Frangi, W. J. Niessen, R. M. Hoogeveen, T. van Walsum, and M. A. Viergever, "Model-based quantitation of 3-D magnetic resonance angiographic images," IEEE Trans. Medical Imaging 18, pp. 946-956, Oct. 1999.

2. T. Boskamp, D. Rinck, F. Link, B. Kümmerlen, G. Stamm, and P. Mildenberger, "New vessel analysis tool for morphometric quantification and visualization of vessels in CT and MR imaging data sets," Radiographics (RSNA) 24(1), pp. 287-297, 2004.

3. J. F. Canny, "A computational approach to edge detection," IEEE Trans. PAMI 8, pp. 679-698, Nov. 1986.

4. M. Hernández-Hoyos, M. Orkisz, P. Puech, C. Mansard-Desbleds, P. Douek, and I. E. Magnin, "Computer-assisted analysis of three-dimensional MR angiograms," Radiographics (RSNA) 22(2), pp. 421-436, 2002.

5. A. Frangi, Three-Dimensional Model-Based Analysis of Vascular and Cardiac Images. PhD thesis, University of Utrecht, The Netherlands, 2001.

6. R. M. Hoogeveen, Vessel Visualization and Quantification by Magnetic Resonance Angiography. PhD thesis, Utrecht University, The Netherlands, Dec. 1998.

7. J. Reinhardt, N.D. d'Souza and E.A. Hoffman, "Accurate measurement of intrathoracic airways," IEEE Trans. Medical Imaging 12, pp. 820-827, Dec. 1997.

8. R. Manniesing, B.K. Velthuis, M.S. van Leeuwen, I.C. van der Schaaf, P.J. van Laar and W.J. Niessen, "Level set based cerebral vasculature segmentation and diameter quantification in CTA," Med.Im.Anal. 10, pp. 200-214, Apr. 2006.

9. P. R. S. Mendonça, D. Padfield, J. Miller and M. Turek, "Bias in the localization of curved edges," in Proc. European Conf. Computer Vision (ECCV), 2, pp. 554-565, 2004.

10. H. Bouma, A. Vilanova, L. J. van Vliet, and F. A. Gerritsen, "Correction for the dislocation of curved surfaces caused by the PSF in 2D and 3D CT images," IEEE Trans. PAMI 27(9), pp. 1501-1507, 2005.

11. A. F. Frangi, W. J. Niessen, P.J. Nederkoorn, O.E.H. Elgersma and M.A. Viergever, "Three-dimensional modelbased stenosis quantification of the carotid arteries from contrast-enhanced MRA," in Proc. MMBIA, pp. 110-118, June 2000 .

12. R. M. Hoogeveen, C. J. G. Bakker, and M. A. Viergever, "Limits to the accuracy of vessel diameter measurement in MR angiography," J. Magnetic Resonance Imaging 8, pp. 1228-1235, 1998.

13. O. Saba, E.A. Hoffman and J.M. Reinhardt, "Maximizing quantitative accuracy of lung airway lumen and wall measures obtained from X-ray CT imaging," J. Applied Physiology 95, pp. 1063-1075, May 2003.

14. D. Marr and E. Hildreth, "Theory of edge detection," in Proc. Royal Society, pp. 187-217, 1980.

15. M. Sonka, V. Hlavac and R. Boyle, Image Proc., Anal. and Machine Vision, Thomson Publishing, USA, 1999.

16. M. Bosma, Iso-Surface Volume Rendering. PhD thesis, University of Twente, Enschede, The Netherlands, 2000.

17. E. Nickoloff and R. Riley, "Simplified approach for modulation transfer function determination in computed tomography," Medical Physics 12, pp. 437-442, July 1985.

18. F. Heijden, Image Based Measurement Systems: Object Recognition and Parameter Estimation, John Wiley and Sons, Chichester, UK, 1994. ISBN 0-471-95062-9.

19. B. Haar Romeny, Front-End Vision and Multi-Scale Image Analysis, Kluwer, The Netherlands, 2003.

20. R. Fahrig, A. J. Fox, and D. W. Holdsworth, "A three-dimensional cerebrovascular flow phantom," Medical Physics 26(8), pp. 1589-1598, 1999.

21. M. Kass and A. Witkin, "Analyzing oriented patterns," Computer Vision, Graphics, and Image Processing 37(3), pp. 362-385, 1987. 